\title{
Mycolicibacterium fortuitum genomic epidemiology, resistome and virulome
}

\author{
Sergio Morgado ${ }^{1 /+}$, Nilcéia de Veiga Ramos² ${ }^{2}$ Fernanda Freitas ${ }^{1}$, \\ Érica Lourenço da Fonseca', Ana Carolina Vicente ${ }^{1 /+}$
}

${ }^{1}$ Fundação Oswaldo Cruz-Fiocruz, Instituto Oswaldo Cruz, Laboratório de Genética Molecular de Microrganismos, Rio de Janeiro, RJ, Brasil ²Universidade de Vassouras, Faculdade de Ciências Médicas de Maricá, Maricá, RJ, Brasil

BACKGROUND Mycolicibacterium fortuitum is an opportunistic pathogen associated with human and animal infection worldwide. Studies concerning this species are mainly represented by case reports, some of them addressing drug susceptibility with a focus on a specific geographic region, so there is a gap in relation to the global epidemiological scenario.

OBJECTIVES We aimed determine the global epidemiological scenario of $M$. fortuitum and analyse its traits associated with pathogenicity.

METHODS Based on publicly available genomes of $M$. fortuitum and a genome from Brazil (this study), we performed a genomic epidemiology analysis and in silico and in vitro characterisation of the resistome and virulome of this species.

FINDINGS Three main clusters were defined, one including isolates from the environment, human and animal infections recovered over nearly a century. An apparent intrinsic resistome comprises mechanisms associated with macrolides, betalactams, aminoglycosides and antitubercular drugs such as rifampin. Besides, the virulome presented Type VII secretion systems (T7SS), including ESX-1, ESX-3, ESX-4 and ESX-4-bis, some of which play a role on the virulence of Mycobacteriaceae species.

MAIN CONCLUSIONS Here, M. fortuitum was revealed as a reservoir of an expressive intrinsic resistome, as well as a virulome that may contribute to its success as a global opportunist pathogen.

Key words: arr - rifampin - resistance - ESX - type VII secretion system - opportunist pathogen

Mycobacteriaceae comprehends a wide spectrum of environmental and pathogenic bacteria that eventually arise in clinics affecting human and animal health. Recently, members of this family have been reclassified into new genera, and thus rapid-growing species, such as Mycobacterium fortuitum, now belong to the Mycolicibacterium genus. ${ }^{(1)}$ Mycolicibacterium fortuitum is ubiquitous in the environment and its role as a pathogen is being recognised worldwide. ${ }^{(2)}$ In fact, the burden of disease due to non-tuberculous mycobacteria (NTM) may be underestimated, as infections by these organisms are not on the reportable list. ${ }^{(3)}$ In addition, NTM infections are increasing globally in humans and animals, and $M$. fortuitum is among the most prevalent NTM species enrolled in this scenario. ${ }^{(4,5,6)} M$. fortuitum infections have been reported with a high prevalence of resistance to several drugs, including macrolides, betalactams, aminoglycosides and tetracyclines, in addition to antitubercular drugs (e.g., isoniazid, rifampin, ethambutol, clofazimine, ethionamide, and rifabutin). ${ }^{(7,8,9)}$ So

doi: 10.1590/0074-02760210247

Financial support: This study was partially supported by the postdoctoral fellowship Inova Fiocruz/Fundação Oswaldo Cruz.

+ Corresponding authors: sergio.morgado@ioc.fiocruz.br / anapaulo@ioc.fiocruz.br (1) https://orcid.org/0000-0003-4877-7639/ https://orcid.org/0000-0001-7086-2042 Received 16 July 2021

Accepted 13 October 2021 far, studies of $M$. fortuitum are mainly represented by case reports in humans and animals, some of them addressing drug susceptibility with a focus on a specific geographic region, so there is a gap in relation to the global epidemiological scenario and systematic analysis of $M$. fortuitum traits associated with pathogenicity. The availability of $M$. fortuitum genomic information and metadata associated with these organisms (e.g., host, geographic location, year) is an opportunity to begin to fill the gap in the general biological characteristics of this zoonotic and widespread opportunistic bacteria.

\section{MATERIALS AND METHODS}

Genome sequences analysed - A total of 25 M. fortuitum genomes were retrieved from the NCBI site (https:// www.ncbi.nlm.nih.gov/genome/browse/\#!/prokaryotes/14575/) in June 2021 (Table).

Genome sequencing and assembly - Genomic DNA of M. fortuitum 7G strain was extracted using NucleoSpin Microbial DNA (Macherey-Nagel) and sequenced with Nextera XT library kit on the Illumina HiSeq 2500 platform, generating $2 \times 250 \mathrm{bp}$ paired-end reads. The raw reads were submitted to quality control by NGS QC Toolkit v2.3.3 ${ }^{(10)}$ and the genome was assembled using SPAdes v3.14.1.11) The genome and the raw reads were deposited at NCBI under the accession numbers JAEQRQ000000000 and SRR15257947, respectively.

Drug susceptibility testing and cloning - M. fortuitum 7G strain was grown in trypticase soy agar (TSA) medium supplemented with $0.05 \%$ Tween-80 (Sigma- 
Aldrich) at $22^{\circ} \mathrm{C}$ for $72 \mathrm{~h}$. Drug susceptibility of the M. fortuitum 7G strain was evaluated by E-test method (bioMerieux) in Mueller-Hinton agar plates for various drugs: azithromycin, clarithromycin, streptomycin, tobramycin, meropenem, cefalotin, cefepime, and rifampicin. The arr and rox polymerase chain reaction (PCR) products were cloned into the pGEM T-Easy Cloning Vector System (Promega) and used to transform Escherichia coli DH5 $\alpha$ lineage (rifampicin MIC of $4 \mu \mathrm{g} / \mathrm{mL}$ ).

Phylogenetic analysis - The M. fortuitum genomes were annotated using Prokka v1.14. $6^{(12)}$ and submitted to Roary v3.13.0 $0^{(13)}$ to determine the core genome. The single-nucleotide polymorphism (SNP) sites of the concatenated core genes $(180,276 \mathrm{bp})$ were obtained using snp-sites v2.5.1.(14) A phylogenetic neighbor joining tree was generated using PhyML v3.1 in Seaview v4 ${ }^{(15)}$ with 1000 bootstrap replicates.

Resistome and virulome analysis - M. fortuitum genomes were surveyed for antibiotic resistance and virulence genes through ABRicate (https://github.com/tseemann/abricate) based on The Comprehensive Antibiotic Resistance Database ${ }^{(16)}$ and Virulence Factor Database. (17) In addition, T7SS was searched based on the identification of the T7SS core proteins ${ }^{(18)}$ using HMMer package v3.1b2..$^{(19)}$

\section{RESULTS AND DISCUSSION}

Here, based on 25 M. fortuitum genomes (drafts and completes) available in the NCBI database and a sequenced genome from Brazil (this study; accession number JAEQRQ000000000), we performed a genomic epidemiology analysis and in silico and in vitro characterisation of the resistome ${ }^{(20)}$ of this species. This is a diverse set of $M$. fortuitum genomes considering the occurrence of the strains in terms of space, time, and origin, since they were isolated from environments, animals, and humans in several countries for almost a century (1923-2020). A core genome SNP analysis generated a neighbor-joining tree that revealed three main clusters (Figure). Based on the available metadata, it was possible to associate clusters I and III with isolates from different countries, while cluster II is composed only of isolates from South Africa. All isolates from cluster I (India, Mozambique, and Cambodia; 2008-2012) and II (South Africa; 2011-2012) were from human infections. Cluster III is quite diverse with isolates from human and animal infections, as well as from the environment, being recovered over almost a century (1923 to 2020) (Table).

The in silico inference of the resistome was performed using CARD, resulting in the identification, in all genomes of clusters I, II and III, of the followed genes: aph (aminoglycoside O-phosphotransferase), aac (aminoglycoside acetyltransferase), arr $\left(\mathrm{NAD}^{+}\right.$rifampin ADP-ribosyltransferase), blaF (beta-lactamase), erm (23S ribosomal RNA methyltransferase), rbpA (RNA polymerase-binding protein), rox (rifampin monooxygenase) and tap (multidrug efflux pump). Each of these genes was found in the same chromosomal location, with no evidence of association with mobile platforms, suggesting the constitutive nature of this resistome in this species. In addition to this in silico analysis and to gain some insights into the functionality of these antibiotic resistance genes (ARGs), we performed in vitro analyses with $M$. fortuitum $7 \mathrm{G}$ strain, defining the minimum inhibitory concentration (MIC) for the antibiotic classes represented in the resistome. The $M$. fortuitum $7 \mathrm{G}$ strain showed high resistance rates to macrolides (azithromy$\operatorname{cin}>256 \mu \mathrm{g} / \mathrm{mL}$ and clarithromycin $>32 \mu \mathrm{g} / \mathrm{mL}$ ), aminoglycosides (streptomycin $>32 \mu \mathrm{g} / \mathrm{mL}$ and tobramycin $>32 \mu \mathrm{g} / \mathrm{mL}$ ), carbapenem (meropenem $>32 \mu \mathrm{g} / \mathrm{mL}$ ), cephalosporins (cefalotin $>256 \mu \mathrm{g} / \mathrm{mL}$ and cefepime $>$ $256 \mu \mathrm{g} / \mathrm{mL}$ ) and rifampicin $>32 \mu \mathrm{g} / \mathrm{mL}$. In fact, Nash et al. ${ }^{(21)}$ observed that $M$. fortuitum strains were naturally resistant to macrolides and that this resistance would be associated with the erm gene. Erm belongs to a diverse family of proteins encoded by a heterogeneity of alleles, some of them (erm37-41) intrinsically associated with Mycobacteriaceae species. Here, erm39 was identified in all $M$. fortuitum genomes and its functionality was demonstrated in M. fortuitum $7 \mathrm{G}$, as it had already been shown in the CT6 strain. ${ }^{(21)}$ The aph(3")-Ic gene was identified for the first time in an environmental $M$. fortuitum strain, being involved in molecular mechanisms of streptomycin resistance in some Mycobacteriaceae and Streptomyces. ${ }^{(22)}$ It is worth noting the identification in all genomes of a set of determinants, related to different resistance mechanisms, associated with the rifamycin class of antibiotics. The $a r r, r b p \mathrm{~A}$, rox and tap genes may impact resistance to rifampicin, ${ }^{(23,24)}$ a firstline drug that has been used to treat Mycobacterium tuberculosis infections for more than half a century. We then experimentally determined the activity of some of these genes (arr and rox) carried by the M. fortuitum 7G strain. Based on arr and rox genes cloning and transformation in heterologous system, it was demonstrated that this arr allele was associated with a high rate of resistance $(32 \mu \mathrm{g} / \mathrm{mL})$, while rox did not improve the $E$. coli rifampicin MIC, indicating that this Arr offers resistance to rifampin for M. fortuitum $7 \mathrm{G}$ strain. Besides the conservative $M$. fortuitum resistome, a class 1 integron carrying a qac/sull gene cassette was identified in a genome (E3337) from cluster III (Table) and, therefore, this would be the first evidence of this genetic element of resistance in Mycolicibacterium. Class 1 integron is a genomic platform in which antibiotic resistance genes are acquired and expressed, contributing to the emergence of resistance in a one-step fashion, ${ }^{(25)}$ therefore, it represents a possibility to increase the resistance spectrum in one generation. In general, in bacteria, plasmids are another genetic element strongly associated with the bacterial resistome, but particularly for $M$. fortuitum, its resistome was entirely associated with the chromosomal genomic context. In fact, a previous survey of $\mathrm{Mycol}-$ icibacterium mobilome showed an $8 \mathrm{~kb}$ non-mobilisable plasmid, without association to any ARG, shared by two M. fortuitum metagenomes (SCH6189132/cluster II/ South Africa and MTB7/cluster III/Morocco). ${ }^{(18)}$

The $M$. fortuitum virulome was accessed using ABRicate based on Virulence Factor Database. Four genes were identified in all genomes: icl, ideR, phoP, and relA (except relA in GA-0871). These genes were associated with 


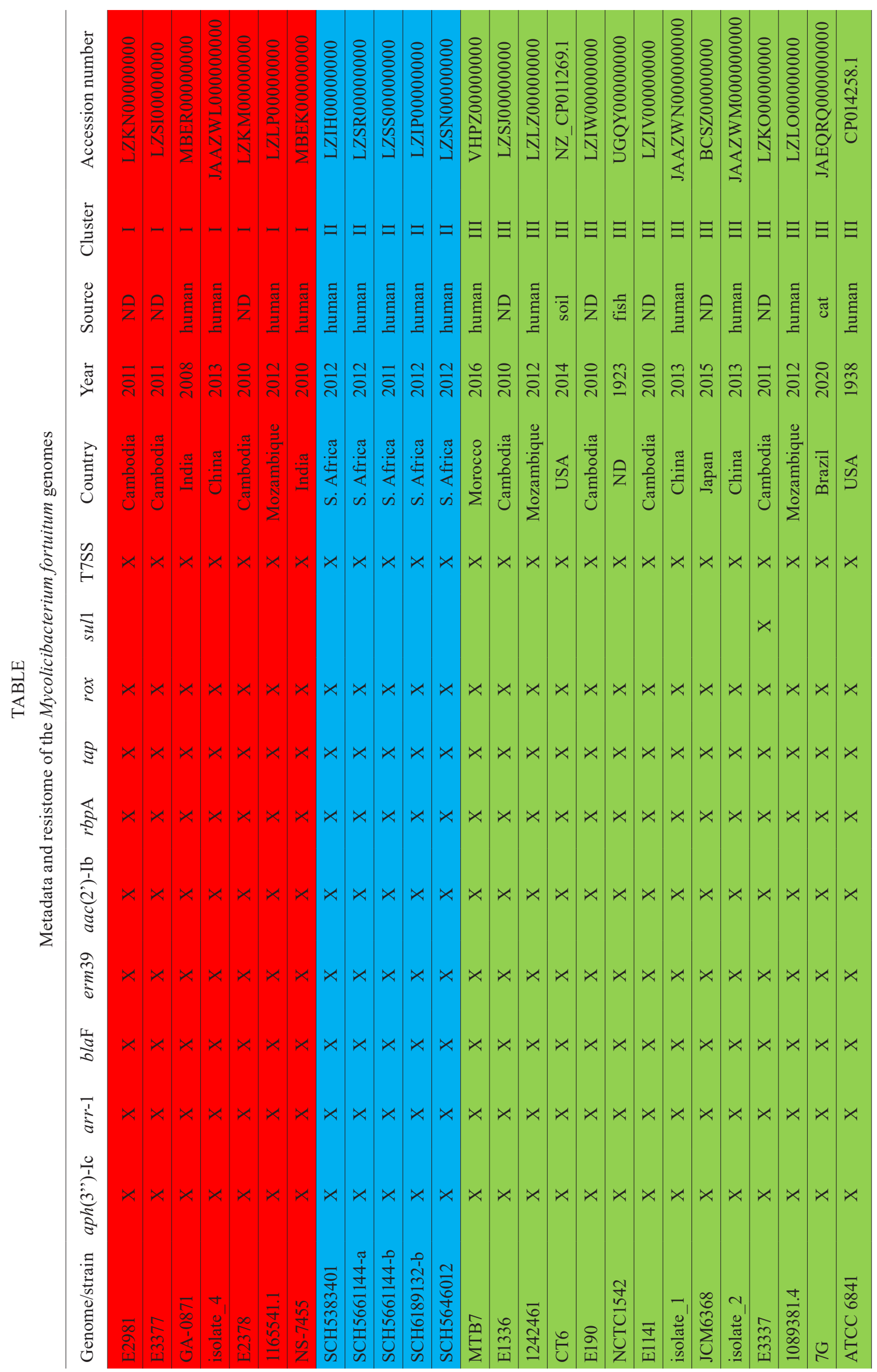




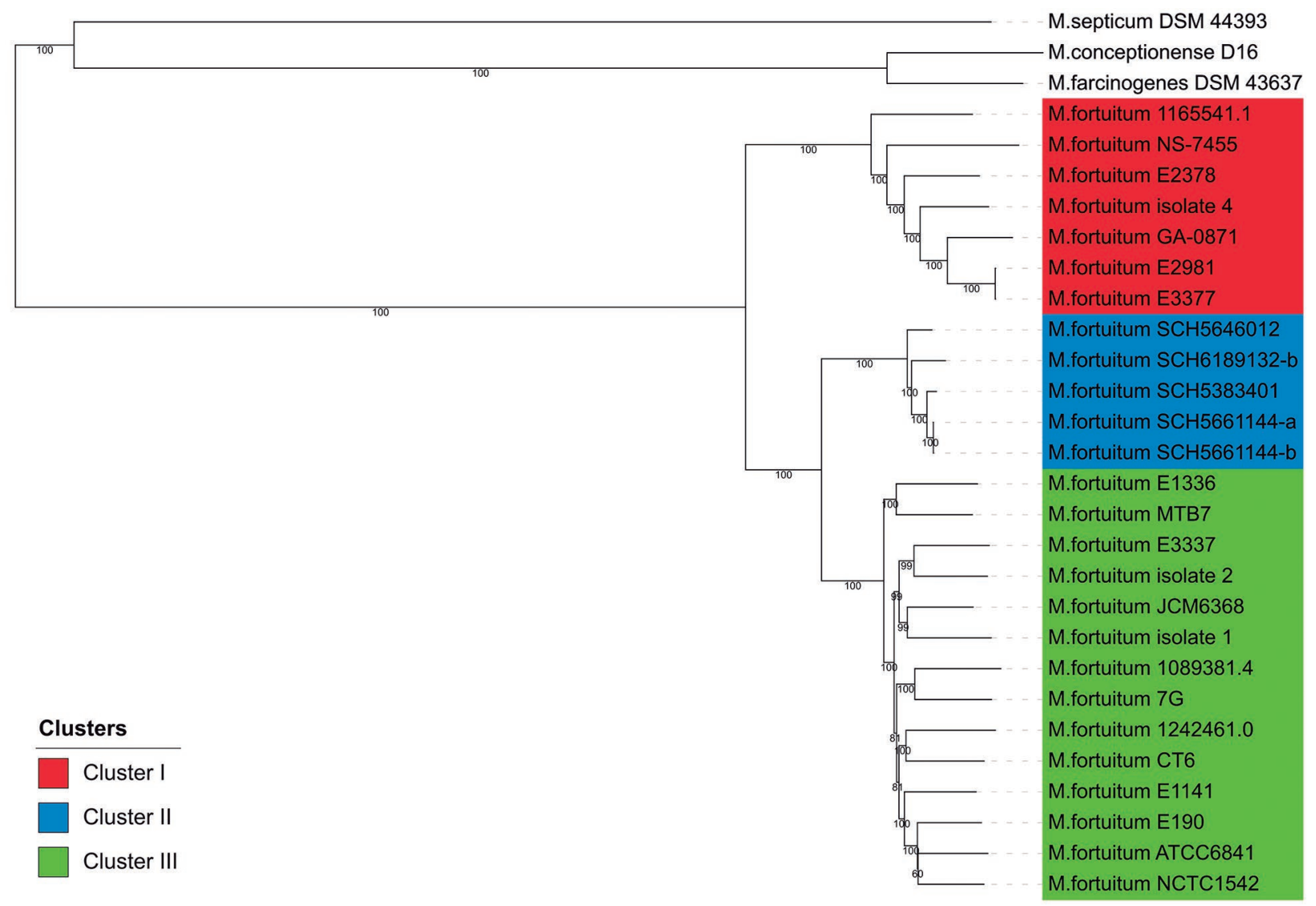

Single-nucleotide polymorphism (SNP)-based tree of 26 Mycolicibacterium fortuitum genomes highlighting the three main clusters.

stress response, persistence, and iron uptake, indirectly impacting the virulence. In addition, we also searched for the T7SS, which is the only specialised secretion system of these organisms. T7SS is encoded by six paralogous chromosomal loci (ESX-1, -2, -3, -4, -4 bis, and -5) and has been associated with several functions, including virulence. ${ }^{(26,27)}$ All M. fortuitum genomes had ESX-1, ESX-3, ESX-4 and ESX-4-bis Type VII secretion systems, some of which play an essential role in Mycobacterium virulence, nutrient uptake and conjugation. ${ }^{(26,27)}$

Here, the ubiquitous M. fortuitum bacterium proved to be a reservoir of an expressive intrinsic resistome and virulome, despite the spatiotemporal diversity of the strains, which indicates a constitutive trait of the species that may contribute to its success as a global opportunist pathogen.

\section{AUTHORS' CONTRIBUTION}

SMM, ELF and ACPV - Conception, design, data analysis and manuscript writing; FFJ and NVR experimental work. The authors declare no conflict of interest.

\section{REFERENCES}

1. Gupta RS, Lo B, Son J. Phylogenomics and comparative genomic studies robustly support division of the genus Mycobacterium into an emended genus Mycobacterium and four novel genera. Front Microbiol. 2018; 9: 67.

2. Halstrom S, Price P, Thomson R. Environmental mycobacteria as a cause of human infection. Int J Mycobacteriol. 2015; 4(2): 81-91.
3. Primm TP, Lucero CA, Falkinham JO. Health impacts of environmental mycobacteria Clin Microbiol Rev. 2004; 7(1): 98-106.

4. Winburn B, Sharman T. Atypical mycobacterial disease. StatPearls [Internet]. Treasure Island (FL): StatPearls Publishing; 2021 Jan. Available from: https://www.ncbi.nlm.nih.gov/books/ NBK430685/.

5. Delghandi MR, El-Matbouli M, Menanteau-Ledouble S. Mycobacteriosis and infections with non-tuberculous mycobacteria in aquatic organisms: a review. Microorganisms. 2020; 8(9): 1368.

6. Shrivastava K, Kumar C, Singh A, Narang A, Giri A, Sharma NK, et al. An overview of pulmonary infections due to rapidly growing mycobacteria in South Asia and impressions from a subtropical region. Int J Mycobacteriol. 2020; 9(1): 62-70.

7. Santos DR, Lourenço MC, Coelho FS, Mello FC, Duarte RS. Resistance profile of strains of Mycobacterium fortuitum isolated from clinical specimens. J Bras Pneumol. 2016; 42(4): 299-301.

8. Shen Y, Wang X, Jin J, Wu J, Zhang X, Chen J, et al. In vitro susceptibility of Mycobacterium abscessus and Mycobacterium fortuitum isolates to 30 antibiotics. Biomed Res Int. 2018; 2018: 4902941.

9. Lin C, Russell C, Soll B, Chow D, Bamrah S, Brostrom R, et al. Increasing prevalence of nontuberculous mycobacteria in respiratory specimens from US-Affiliated Pacific Island Jurisdictions. Emerg Infect Dis. 2018; 24(3): 485-91.

10. Patel RK, Jain M. NGS QC toolkit: a toolkit for quality control of next generation sequencing data. PLoS One. 2012; 7(2): e30619.

11. Bankevich A, Nurk S, Antipov D, Gurevich AA, Dvorkin M, Kulikov AS, et al. SPAdes: a new genome assembly algorithm and 
its applications to single-cell sequencing. J Comput Biol. 2012; 19(5): 455-77.

12. Seemann T. Prokka: rapid prokaryotic genome annotation. Bioinformatics. 2014; 30(14): 2068-9.

13. Page AJ, Cummins CA, Hunt M, Wong VK, Reuter S, Holden MT, et al. Roary: rapid large-scale prokaryote pan genome analysis. Bioinformatics. 2015; 31(22): 3691-3.

14. Page AJ, Taylor B, Delaney AJ, Soares J, Seemann T, Keane JA, et al. SNP-sites: rapid efficient extraction of SNPs from multiFASTA alignments. Microb Genom. 2016; 2(4): e000056.

15. Gouy M, Guindon S, Gascuel O. SeaView version 4: a multiplatform graphical user interface for sequence alignment and phylogenetic tree building. Mol Biol Evol. 2010; 27(2): 221-4.

16. Alcock BP, Raphenya AR, Lau T, Tsang KK, Bouchard M, Edalatmand A, et al. CARD 2020: antibiotic resistome surveillance with the comprehensive antibiotic resistance database. Nucleic Acids Res. 2020; 48(D1): D517-25.

17. Chen L, Yang J, Yu J, Yao Z, Sun L, Shen Y, et al. VFDB: a reference database for bacterial virulence factors. Nucleic Acids Res. 2005; 33: D325-8.

18. Morgado SM, Vicente ACP. Comprehensive in silico survey of the Mycolicibacterium mobilome reveals an as yet underexplored diversity. Microb Genom. 2021; 7(3): 533.

19. Eddy SR. Accelerated profile HMM searches. PLoS Comp Biol. 2011; 7: e1002195.
20. Fonseca EL, Andrade BGN, Vicente ACP. The resistome of lowimpacted marine environments is composed by distant metallo- $\beta$ lactamases homologs. Front Microbiol. 2018; 5(9): 677.

21. Nash KA, Zhang Y, Brown-Elliott BA, Wallace Jr RJ. Molecular basis of intrinsic macrolide resistance in clinical isolates of $\mathrm{Myco}$ bacterium fortuitum. J Antimicrob Chemother. 2005; 55(2): 170-7.

22. Ramón-García S, Otal I, Martín C, Gómez-Lus R, Aínsa JA. Novel streptomycin resistance gene from Mycobacterium fortuitum. Antimicrob Agents Chemother. 2006; 50: 3920-2.

23. Koteva K, Cox G, Kelso JK, Surette MD, Zubyk HL, Ejim L, et al. Rox, a rifamycin resistance enzyme with an unprecedented mechanism of action. Cell Chem Biol. 2018; 25(4): 403-12.

24. da Fonseca EL, Freitas F, de Amorim JC, Vicente AC. Detection of new arr- 4 and arr-5 gene cassettes in clinical Pseudomonas aeruginosa and Klebsiella pneumoniae strains from Brazil. Antimicrob Agents Chemother. 2008; 52(5): 1865-7.

25. Cambray G, Guerout A, Mazel D. Integrons. Annu Rev Genet. 2010; 44: 141-66.

26. Lagune M, Petit C, Sotomayor FV, Johansen MD, Beckham KSH, Ritter C, et al. Conserved and specialized functions of Type VII secretion systems in non-tuberculous mycobacteria. Microbiology. 2021; 167: 1054

27. Mortimer TD, Weber AM, Pepperell CS. Evolutionary thrift: mycobacteria repurpose plasmid diversity during adaptation of type VII secretion systems. Genome Biol Evol. 2017; 9(3): 398-413. 